\title{
La asistencia a pacientes crónicos y pluripatológicos. Magnitud e iniciativas para su manejo: La Declaración de Sevilla. Situación y propuestas en Galicia

\author{
The chronic patient with plurypathology. Magnitude and initiatives for
} management: The Seville Statement. Situation and proposals in Galicia
}

\author{
Julio Montes Santiago ${ }^{1}$, Emilio Casariego Vales², Manuel de Toro Santos ${ }^{3}$, Esther Mosquera ${ }^{4}$ \\ ${ }^{1}$ Servicio de Medicina Interna. Complejo Hospitalario Universitario de Vigo (CHUVI). SERGAS. Vigo \\ ${ }^{2}$ Servicio de Medicina Interna. Hospital Universitario Lucus Augusti. SERGAS. Lugo \\ ${ }^{3}$ Servicio de Medicina Interna. Complexo Hospitalario Universitario de Ourense (CHOU). SERGAS. Ourense \\ ${ }^{4}$ Gerente regional de Programas de Gestión Sanitaria. AstraZeneca
}

\section{Resumen}

El progresivo envejecimiento de las poblaciones con el exponencial crecimiento de pacientes con enfermedades crónicas y pluripatológicas y dependencia que ello conlleva ha supuesto importantes desafíos para las administraciones y financiación de los sistemas sanitarios. Por ello, estos están desarrollando diversas estrategias para afrontar tales desafíos. También en España la Declaración de Sevilla 2011 ha impulsado un Plan integral nacional sobre atención al paciente con enfermedades crónicas, basado en tres pilares: involucrar al paciente y la comunidad, establecer pautas para organizar la asistencia y desarrollar la información clínica y toma de decisiones. Ello es particularmente importante en Galicia que presenta una población más envejecida y con mayor prevalencia de enfermedades crónicas que la media nacional. En tal sentido la Estrategia SERGAS 2014 en dicha Comunidad pretende aportar objetivos y líneas de actuación para enfrentar tales problemas. En sintonía con tales planes en nuestro entorno la SOGAMI ha establecido un plan de inicio para involucrar a sus miembros en una atención más informada y eficiente para los pacientes crónicos y pluripatológicos.

PALABRAS CLAVE: Pacientes pluripatológicos. Declaración de Sevilla. Sociedad Gallega de Medicina interna.

\section{Dimensión del problema: envejecimiento, enfermedades crónicas, pluripatología y dependencia}

En las últimas décadas estamos asistiendo a un envejecimiento progresivo de las poblaciones. En la UE el porcentaje de personas > 65 años pasará del 16,1\% (2000) al 27,5\% (2050). España, junto a Italia y Japón, están a la cabeza de este proceso de envejecimiento mundial, estimándose que para esas fechas cerca del 35\% de nuestra población superará los 65 años. Una de las consecuencias inmediatas de este efecto es el rápido aumento de pacientes con múltiples enfermedades crónicas. En España la Encuesta Nacional de Salud (2006) mostró una media de 2,8 problemas -entre 65-74 años- y de 3,2 -en >75 años. Además de la avanzada edad y el género femenino otras circunstancias que incrementan la posibilidad de padecer procesos pluripatológicos son la obesidad, los bajos niveles socioeconómicos o culturales, el vivir sólo o residir en instituciones para ancianos ${ }^{1-5}$.

\begin{abstract}
The progressive aging of populations with an exponential growth of patients with chronic and polypathologic diseases and associated disabilities pose significant challenges for Administration and Finances of the Health systems. Therefore, some strategies to address these problems are developing. In Spain, the "Declaración de Sevilla 2011" (Sevilla statement) is impulsing a national comprehensive plan care for patients with chronic diseases, based on three fundaments: involvement of the patient and community, establishment of guidelines to organize care and development of clinical information and decision making. These are particularly important because in Galicia lives an extremely aged population with increased prevalence of chronic diseases. In this sense, the objective of "SERGAS 2014" proposal is to provide aims and lines of action to confront such challenges. In agreement with these plans in our environment, the SOGAMI is establishing an initial plan to involve its members in a more informed and efficient care for patients with chronic and multiple diseases. KEY WORDS: Patients with Polypathology. Sevilla Statement 2011. Galician Society of Internal Medicine.
\end{abstract}

El Informe de la OMS Previniendo las enfermedades crónicas estimó que el $60 \%$ de las muertes mundiales en 2005 , al menos en países desarrollados, eran ya debidas a enfermedades crónicas, si bien tal problema a menudo es infraestimado. Además, la OMS cifra en 650 millones -un 10\% de la población- las personas que sobreviven con discapacidades. Peor aún es que para 2020 estas enfermedades crónicas serán la principal causa de discapacidad y que en 2030 se doblará la incidencia actual de enfermedades crónicas en $>65$ años ${ }^{3}$. P. ej. en España se calcula que en 2050 el 27\% del total de población serán pacientes con problemas de cronicidad. Además tal sector de mayores de 65 años dada esta alta prevalencia de enfermedades precisa de muchos más cuidados y consume más recursos sanitarios (p.ej. el gasto sanitario atribuible a este sector poblacional se ha estimado en 4-12 veces superior al correspondiente a personas de menos edad ${ }^{3}$ ).

La evolución de las enfermedades es causa de disfunciones en los distintos órganos y sistemas, provocando limitaciones 
físicas y psíquicas progresivas que dificultan la realización de las actividades básicas de la vida diaria y determinan la necesidad de ser ayudadas en estas tareas vitales elementales. De hecho, cerca del 2,5\% de la población mundial presenta dependencia debido a enfermedades crónicas ${ }^{3}$. En España este fenómeno es aún más llamativo y se ha calculado que, hasta su muerte, los hombres tendrán como expectativa una media de 7 años incapacitados, que será de 11 para las mujeres ${ }^{4}$.

Mucha de esta necesidad de ayuda es soportada actualmente por los familiares más próximos, pero se observa actualmente una transferencia de estas formas tradicionales de cuidados hacia los servicios de salud y sociales, lo cual hace que los costes previstos de la atención aumenten en próximos años. Se estima que el paciente crónico complejo y/o frágil supone un $5 \%$ de la población y consume el $65 \%$ de la totalidad de recursos sanitarios. Es razonable, pues, pensar que buena parte de la sostenibilidad del Sistema Nacional de Salud (SNS) gravitará en gran parte sobre la gestión de la atención a estos pacientes y motive el desarrollo de planes específicos de atención a los mismos ${ }^{1,2,5,6}$. Y también que deban redefinirse objetivos para las prioridades económicas pues p.ej. en España el gasto público en cuidados de largo plazo es sólo del $0,65 \%$ del PIB frente al $1,39 \%$ de media en la OCDE (Cf. Diario Médico, 6/02/2012).

\section{Definición y características de los pacientes con pluripatología}

Se ha comunicado una amplia variabilidad en la prevalencia de pluripatología entre pacientes con enfermedades crónicas, pero en países desarrollados una aproximación realista es que las personas con múltiples patologías representan $>50 \%$ de la población con enfermedades crónicas. Características destacadas de las personas con estas enfermedades son, ${ }^{6,7}$ : 1. Superar los 65 años y con frecuencia los 80. 2. Padecer varias enfermedades al mismo tiempo. 3. Disminución gradual de su autonomía personal y de la capacidad funcional con importantes repercusiones sociales, familiares y emocionales. 4. Respecto a su relación con el sistema sanitario presentan: a) Seguimiento en múltiples consultas tanto en Primaria como Especializada, generalmente con escasa coordinación, y sujetos a múltiples pruebas diagnósticas, a menudo repetitivas. b) Frecuente uso de los recursos sanitarios de asistencia y de los dispositivos de urgencias. c) Ingresos hospitalarios múltiples 0 atención sanitaria por reagudización de sus enfermedades crónicas con estancias a menudo prolongadas d) Tratamientos plurifarmacológicos con bajo nivel de cumplimiento. e) Importante componente socieconómico añadido que a menudo dificulta esta atención hospitalaria.

Pues bien, para designar a tal tipo de de pacientes, cada vez más frecuentes en las consultas de Atención Primaria y en
Ios servicios hospitalarios, se ha consolidado el término de pacientes pluripatológicos (PPL). De hecho, la prevalencia de estos llega a situarse entre el $21-72 \%$ de los pacientes ingresados en Servicios de Medicina Interna, con una edad cercana a los 70 años. Estos pacientes suponen 20-40\% de los ingresos hospitalarios (40,5\% $\geq$ de 3 ingresos/año; 13\% $\geq 5$ ingresos/año). El 10\% de pacientes crónicos ocupan el $55 \%$ de los días de hospitalización y un 5\% de ellos el $42 \%$. En Atención Primaria constituyen aproximadamente el 2-5 $\%$ de la población usuaria de los centros de salud, pero con un consumo de recursos (frecuentación, visitas domiciliarias, pruebas complementarias y farmacia) que cuadruplica el promedio por paciente y presentan un grado de complejidad clínica que requiere múltiples contactos con el medio hospitalario ${ }^{8-11}$. Por ello, se ha insistido en la necesidad de prestarles una atención sustentada en la continuidad asistencial con estrecha coordinación entre Primaria y Hospitalaria, y de ambas con los Servicios sociales.

Desde hace tiempo y en las bases administrativas, el concepto de paciente pluripatológico es conocido y se ha buscado definir mediante instrumentos como el ampliamente utilizado índice de comorbilidad de Charlson, o se han intentado introducir escalas de estratificación con interés pronóstico [Escala CIRS -Chronic IIIness Resources Survey-, ICED index -Index of Coexisting Disease-, índice de Kaplan-Feinstein, etc, que han sido objeto de revisión recientes $\left.{ }^{3,12}\right]$. El inconveniente de tales índices es que no valoran bien el deterioro funcional 0 resulta muchas veces difícil la decisión sobre cuáles de los múltiples procesos que presenta el paciente son los principales responsable de su deterioro.

Ello ha motivado que se careciera de una definición unánime de tales pacientes, que permitieran una homogeneidad en su manejo y se pudieran comparar y evaluar estrategias adecuadas para el mismo. Por ello, en el año 2002 un grupo de trabajo perteneciente a la Sociedad Andaluza de Medicina Interna propuso unos criterios operativos de paciente pluripatológico. Dichos criterios modificados y actualizados en 2007 y auspiciados por la Sociedad Española de Medicina Interna (SEMI) han ido ganando amplia aceptación y se reflejan en la Tabla $1^{13}$. El establecimiento de dichos criterios ha facilitado incluso la elaboración de índices como el PROFUND y PROFUNCTION Simplificado -que disponibles incluso en aplicación informática (CalculaPROFUND.exe)- permiten aproximar a 12 meses un pronóstico de mortalidad y capacidad funcional para aquilatar mejor las necesidades y modo de atención ${ }^{14}$.

El incremento de demanda para este tipo de pacientes ha ocasionado que los hospitales deban replantearse el tipo de asistencia clásica basada en la organización de los cuidados en unidades médicas con el único propósito de manejarlos de forma puntual a un modelo que contemple la continuidad de organización asistencial. Las experiencias han sido diferentes 
Tabla 1. Criterios o categorías definitorias de paciente pluripatológico (PPP)

\begin{tabular}{|c|c|}
\hline Categoría A & $\begin{array}{l}\text { A.1. Insuficiencia cardiaca que en situación de estabilidad clínica haya estado en grado II de la New York Heart Association (Ligera } \\
\text { limitación de la actividad física. La actividad física habitual le produce disnea, angina, cansancio o palpitaciones). } \\
\text { A.2. Cardiopatía isquémica (angina o infarto) }\end{array}$ \\
\hline Categoría B & $\begin{array}{l}\text { B.1. Vasculitis y enfermedades autoinmunes sistémicas } \\
\text { B.2. Enfermedad renal crónica definida por elevacion de } \mathrm{Cr}(>1,4 \mathrm{mg} / \mathrm{dl} \text { en varones; }>1,3 \mathrm{mg} / \mathrm{dl} \text { en mujeres) o proteinuriab mantenidos } \\
\text { durante } 3 \text { meses (Indice albumina/creatinina }>300 \mathrm{mg} / \mathrm{g} \text {, microalbuminuria }>3 \mathrm{mg} / \mathrm{dl} \text { en muestra de orina } 0 \text { albúmina }>300 \mathrm{mg} / \\
\text { día en orina de } 24 \mathrm{~h} 0>200 \mathrm{~g} / \mathrm{min} \text { ) }\end{array}$ \\
\hline Categoría C & $\begin{array}{l}\text { C.1. Enfermedad respiratoria crónica que en situacion de estabilidad clinica haya estado con disnea grado } 2 \text { de la Medical Research } \\
\text { Council (MRC) (Incapacidad de mantener el paso de otra persona de la misma edad caminando en llano debido a la dificultad } \\
\text { respiratoria o tener que parar a descansar al andar en llano al propio paso).o volumen expiratorio forzado en un segundo (FEV1) } \\
<65 \%, 0 \mathrm{SaO} \leq 90 \%\end{array}$ \\
\hline Categoría D & $\begin{array}{l}\text { D.1. Enfermedad inflamatoria crónica intestinal } \\
\text { D.2. Hepatopatía crónica con datos de insuficiencia hepatocelular INR (International Normatized Ratio) }>1,7 \text {; albúmina }<3,5 \mathrm{~g} / \mathrm{dl} \text {; } \\
\text { bilirrubina }>2 \mathrm{mg} / \mathrm{dl} \text { ). } 0 \text { hipertensión portal (Definida por la presencia de datos clínicos, analíticos, ecográficos } 0 \text { endoscópicos }\end{array}$ \\
\hline Categoría E & $\begin{array}{l}\text { E.1. Ataque cerebrovascular } \\
\text { E.2. Enfermedad neurológica con déficit motor permanente que provoque una limitación para las actividades básicas de la vida diaria } \\
\text { (Indice de Barthel }<60 \text { ) } \\
\text { E.3. Enfermedad neurológica con deterioro cognitivo permanente, al menos moderado (Pfeiffer con } \geq 5 \text { errores) }\end{array}$ \\
\hline Categoría F & $\begin{array}{l}\text { F.1. Arteriopatía periférica sintomática } \\
\text { F.2. Diabetes mellitus con retinopatía proliferativa o neuropatía sintomática }\end{array}$ \\
\hline Categoría G & $\begin{array}{l}\text { G.1. Anemia crónica por pérdidas digestivas o hemopatía adquirida no subsidiaria de tratamiento curativo que presente hemoglobina } \\
<10 \mathrm{mg} / \mathrm{dl} \text { en dos determinaciones separadas entre sí mas de } 3 \text { meses } \\
\text { G.2. Neoplasia sólida o hematológica activa no subsidiaria de tratamiento con intención curativa }\end{array}$ \\
\hline Categoría H & $\begin{array}{l}\text { H.1. Enfermedad osteoarticular crónica que provoque por sí misma una limitación para las actividades básicas de la vida diaria (Indice } \\
\text { de Barthel <60) }\end{array}$ \\
\hline
\end{tabular}

Se considera PPP si tiene un criterio de al menos dos categorías diferentes

en cada entorno sanitario, pero características comunes han sido la potenciación de unidades multidisciplinarias y polivalentes con el intento de dar respuesta integral a la descompensación simultánea de las múltiples enfermedades crónicas que padecen. Se han desarrollado así secciones específicas dentro de los servicios de Medicina interna (Unidad de pacientes pluripatológicos, UCEM), consultas de coordinación de Especializada con primaria, potenciación de las Unidades de Hospitalización a domicilio, unidades médicas de corta estancia, hospitales de día, consultas de alta resolución, etc., todo ello con el objetivo de evitar o acortar en lo posible el ingreso en plantas tradicionales de hospitalización y focalizar la atención en la mayor autonomía del paciente.

\section{Planes de actuación. La aplicación en España}

Se ha mostrado posible una mejor atención a los pacientes con enfermedades crónicas, de forma que esta sea más accesible, más eficiente, más aceptable y al mismo tiempo más económica. Así lo has mostrado distintas estrategias surgidas para abordar este problema. Los sistemas de integración más conocidos para la atención a pacientes crónicos son el Kaiser Permanent o el Chronic Care Model (CCM) $)^{15-16}$.

El Kaiser Permanent (el modelo más conocido en nuestro país según una encuesta entre varias Sociedades científicas) ${ }^{16}$ identifica tres niveles de intervención según el grado de complejidad del caso, con el fin de utilizar mejor los recursos humanos y organización asistencial, evitando ingresos hospitalarios y costes innecesarios (Fig. 1). El Modelo de Atención a Enfermedades Crónicas (CCM) es un modelo organizativo basado en la mejora en la coordinación de la atención a los enfermos crónicos, con la finalidad de obtener mejores resultados clínicos y funcionales (Fig. 1). Iniciado hace más de 20 años en el MacColl Institute for Healthcare Innovation de Seattle (EE.UU.) ha sido adaptado a múltiples realidades y países. Incluso la Organización Mundial de la Salud ha denominado a una adaptación del mismo "Modelo de Atención Innovadora a Condiciones Crónicas" (Innovative Care for Chronic Conditions Framework, ICCC) ${ }^{17}$ (Fig. 1). El ICCC incorpora un enfoque poblacional, la coordinación e integración de cuidados, énfasis en la prevención, en la calidad de la atención, la flexibilidad y la toma de decisiones basadas en la evidencia. Pues bien, en el CCM se identifican 6 áreas claves y al implantarlo han de realizarse intervenciones sobre todos los elementos que lo componen de forma simultánea. Estas áreas son: la comunidad, el apoyo en autocuidado, el sistema sanitario, la provisión de servicios, el apoyo en la toma de decisiones y los sistemas de información clínica. La atención a pacientes crónicos discurre en 3 planos superpuestos: a) el conjunto de la comunidad, con sus políticas y múltiples recursos públicos y privados; b) el sistema de salud, con sus organizaciones proveedoras y esquemas de aseguramiento y c) el nivel de la práctica clínica. Todo ello ha de expresarse en la atención sanitaria cotidiana 
Figura 1. Modelos de asistencias a paciente pluripatológicos

Modelo Kaiser permanent

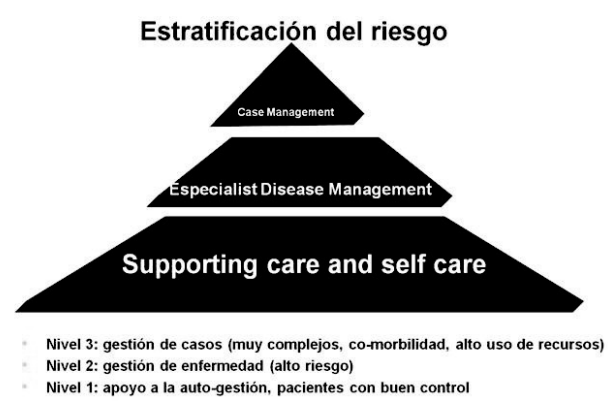

Modelo de Atención a enfermedades crónicas (CCM)

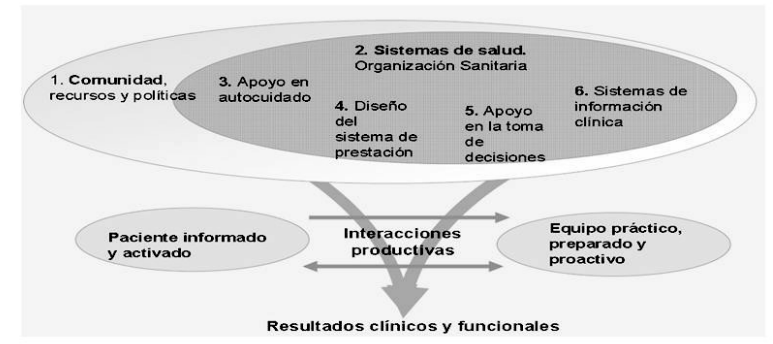

Atención innovadora a las enfermedades crónicas (ICCC, OMS 2002)

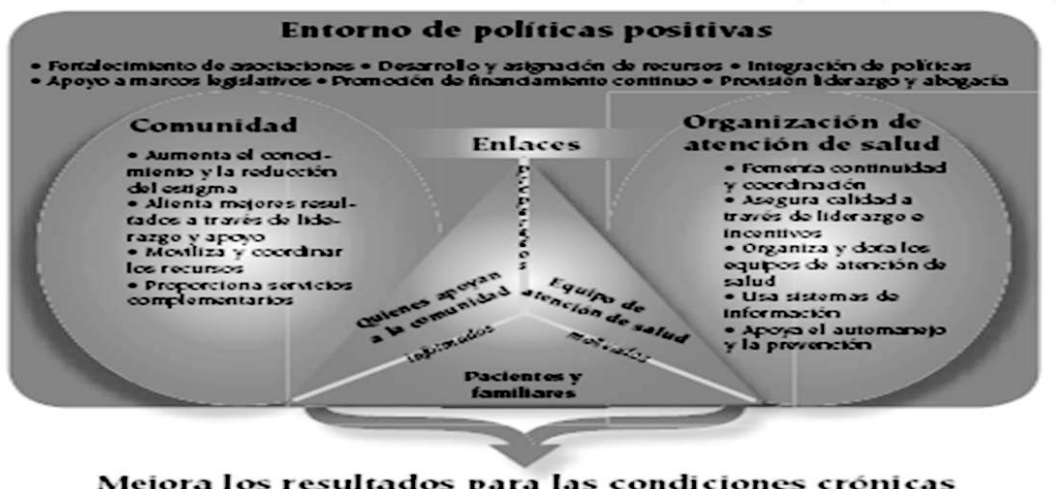

Mejora los resultados para las condiciones crónicas

permitiendo la generación de interacciones productivas entre un paciente informado, activo y comprometido con un equipo práctico, preparado y proactivo.

Aunque en España diversas CCAA (Cataluña, Aragón, Baleares, Valencia, Andalucía, País Vasco, etc.), tomando como paradigma dichos modelos, desarrollaron diversas políticas para la atención de estos pacientes crónicos, en su aspiración de coordinar estas diversas iniciativas un grupo de trabajo de la Sociedad Española de Medicina Interna y de la Sociedad Española de Medicina Familiar y Comunitaria presentó en enero de 2011 un Documento de Consenso para la Atención al Paciente con Enfermedades Crónicas ${ }^{18}$. Ello se fraguó en el contexto de los diversos Congresos de Atención al Paciente Crónico, desarrollados bajo los auspicios de varias Consejerías de Salud de las CCAA. Pues bien al documento mencionado todas las personas 0 asociaciones interesadas pudieron incorporar sugerencias y aportaciones a través de la Web del Observatorio de Prácticas Innovadoras en el Manejo de Enfermedades Crónicas Complejas (OPIMEC) ${ }^{19}$, antes de alcanzar la versión definitiva que se presentó en el III Congreso Nacional de Atención al Paciente Crónico. Dicho Documento de consenso contó con el apoyo de las Consejerías de Salud de todas las CCAA y se ha divulgado a través de los cauces científicos de ambas sociedades. Tal documento de Consenso define explícitamente como su objetivo "promover y estimular el desarrollo de iniciativas en todas las CCAA, coordinadas desde las Gerencias de los Servicios de Salud en colaboración con representantes de la Sociedades Científicas implicadas y de los pacientes, que puedan verse reflejadas en un Plan Integral Autonómico de Atención a los Pacientes con Patologías Crónicas en cada CA, dentro de una estrategia nacional integrada". El documento, tras ponderar los cambios demográficos, la importancia de las enfermedades crónicas, su elevada morbimortalidad, la considerable trascendencia socioeconómica, y las carencias de nuestro sistema sanitario, propugna la necesidad de nuevos modelos organizativos para afrontar la atención a las enfermedades crónicas, proponiendo desarrollar una serie de acciones: la atención debe ser integral, integrada, accesible, longitudinal, activa, participativa, en la educación para la salud y en la gestión clínica por procesos; compete fundamentalmente a médicos de familia, enfermería e internistas; como pilares básicos se debería apoyar en el papel de la Atención Primaria como puerta de entrada al sistema, la historia clínica integrada y sistemas de información sanitaria con indicadores de evaluación adecuados que midan la aceptabilidad, la accesibilidad, la capacidad de identificar y afrontar las necesidades sanitarias de la población, y la adecuada utilización de los recursos.

Una síntesis de tal documento, que en su versión definitiva ha contado con el apoyo de 16 Sociedades Científicas, los Servicios de Salud de la 17 CCAA, el Ministerio de Sanidad y Política Social e Igualdad y el Foro de Pacientes, ha sido denominado Declaración de Sevilla ${ }^{20}$. 
Figura 2. Altas hospitalarias por enfermedades en Galicia (Encuesta Morbilidad Hospitalaria, 2010. Fuente: Instituto Galego de Estadística, 2012

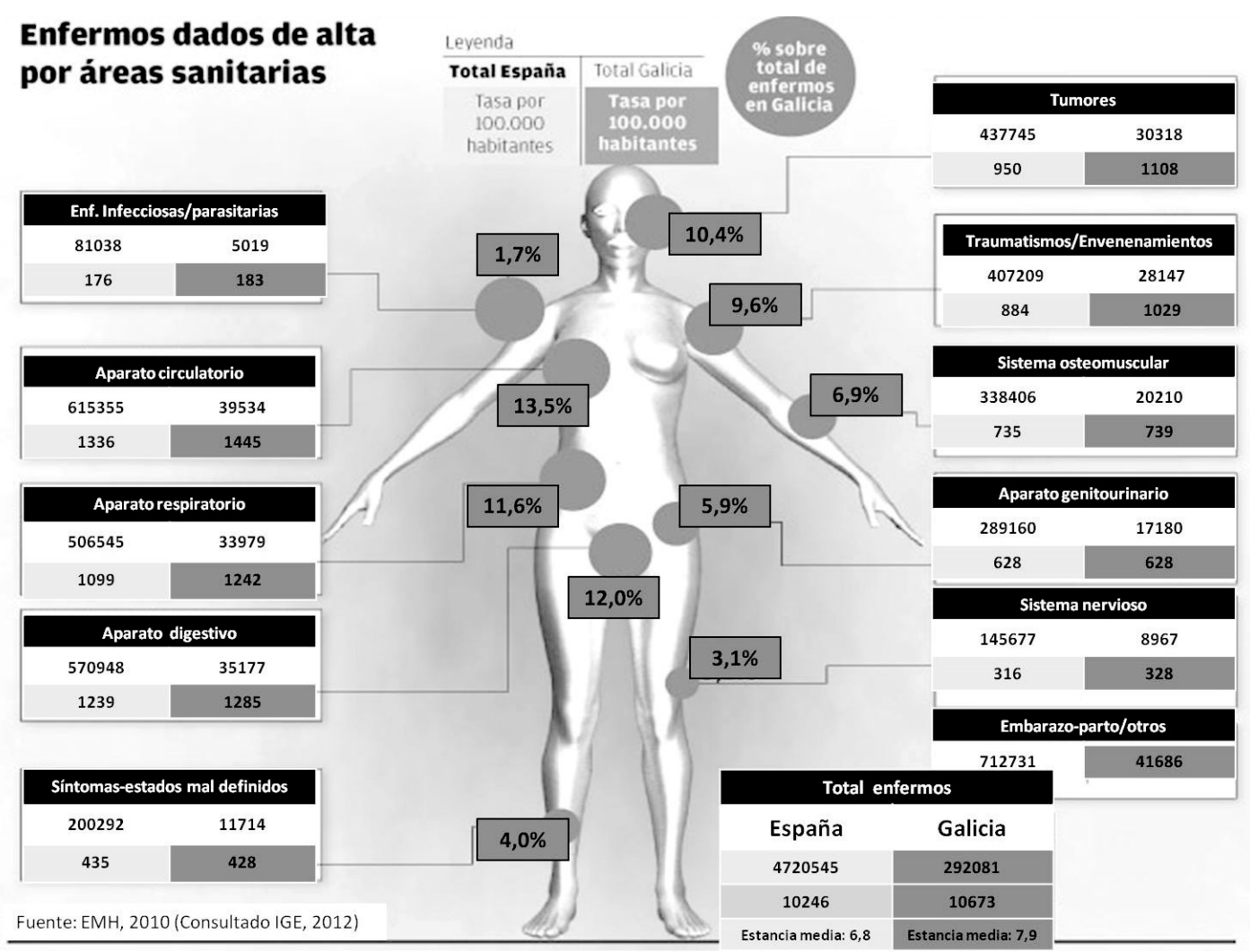

\section{Declaración de Sevilla para la atención} a pacientes con enfermedades crónicas ${ }^{20}$

Profundizando más en dicho documento, que constituye una hoja de ruta para el desarrollo de esta reforma de la asistencia en los próximos años, este se divide en los siguientes apartados:

\section{El paciente y la comunidad}

a) Se declara explícitamente la necesidad de una acción política dirigida a la cronicidad en todas las CCAA. Debe identificarse a las personas vulnerables -empleando la afortunada metáfora acuñada de que el paciente permanezca siempre en la pantalla del radar del sistema sanitario-, así como estratificarse según su nivel de riesgo.

b) Deben estimularse actividades de prevención y de promoción de salud poblacionales e individuales, evaluables, coste-efectivas y con similar nivel de intensidad que el resto de las intervenciones.

c) Es preciso integrar todos los recursos de la comunidad en este objetivo de mejora de salud y de cuidados (Instituciones Públicas y Privadas, Sistema Sanitario, Red Sociosanitaria, Sociedades Científicas, Asociaciones de Pacientes Crónicos, Voluntariado, Bancos del Tiempo, Farmacias Comunitarias) mediante su cooperación coordinada y estableciendo convenios de colaboración y de complementariedad de servicios.

d) Se debe facilitar el autocuidado y adoptar medidas de apoyo a familia y cuidadores. e) Es necesario fortalecer el papel de la enfermería en la prevención y seguimiento.

f) Deben potenciarse los foros de relación, comunicación y colaboración de pacientes y profesionales y de páginas web de calidad en la red.

\section{Organización de la atención}

a) Resaltar a la Atención Primaria como eje coordinador de Ios servicios sanitarios y sociales. Es preciso reforzar el papel del médico y enfermera de familia como agentes principales del paciente y puerta de entrada al sistema, mejorando su capacidad clínica y sus herramientas para la gestión integrada del paciente.

b) Es imprescindible superar la división entre las Asistencias Primaria y Hospitalaria y crear un marco de atención compartida entre los diferentes entornos asistenciales.

c) La reingeniería y gestión por procesos clínicos integrados deben reordenar los servicios para establecer la atención compartida entre los diferentes ámbitos asistenciales.

d) Ha de estratificarse a la población para identificar a pacientes pluripatológicos, frágiles 0 con enfermedades avanzadas y desarrollar planes específicos de atención continuada 0 gestión de casos.

e) Es necesario reforzar el papel de los profesionales con capacidad integradora. La alianza de los médicos y enfermeras de familia, con los médicos internistas y enfermeras hospitalarias, es alternativa innovadora que potencia la 
continuidad asistencial y la personalización de la asistencia sanitaria. A este esquema de asistencia deben incorporarse otros profesionales como farmacéuticos, trabajadores sociales, etc.

f) En esta reorganización de la asistencia el médico de Atención primaria debe ejercer un papel de eje coordinador de los recursos sanitarios con el fin de que la población pueda acceder de forma ordenada a los cuidados sanitarios y sociales. Su actuación, además de clínica debe contemplar problemas sociales relacionados con la dependencia.

g) El internista debe tomar gran relevancia en la atención de pacientes con enfermedades crónicas complejas a través del papel definido como de inter-especialista, enfatizando su aporte de conexión, integración y racionalidad al paciente con comorbilidad y pluripatología.

\section{Información clínica y toma de decisiones.}

Ha de proveerse a los sistemas de información clínica con las condiciones imprescindibles para que sean instrumentos válidos a clínicos y gestores en la toma de decisiones: utilidad, posibilidad de evaluación y que faciliten la autonomía del paciente. En este sentido instrumentos modernos aportados por las tecnologías de la información y comunicación son:

a) Historia clínica electrónica unificada que permita la comunicación segura entre profesionales. Debe incorporar elementos de ayuda en la toma de decisiones, de integración de tratamientos y protección de la seguridad clínica (interacciones, duplicidades o contraindicaciones farmacológicas). Para ello ha de ser consensuada entre los profesionales y Sociedades científicas para que proporciones información relevante tanto a clínicos como gestores. Además debe registrar actividades preventivas y determinantes sociales y ambientales de la enfermedad crónica.

b) Indices clínicos evaluables acordados con los profesionales que permitan realizar un seguimiento del manejo y que permitan establecer objetivos de calidad asistencial, mediante la incentivación del su cumplimiento.

c) Nuevas estrategias de relación entre profesionales y pacientes, mediante el uso de nuevas tecnologías. Deben basarse en el impulso a los autocuidados en un paciente más informado y con mayor autonomía.

Resulta trascendental difundir y apoyar, desde los distintos ámbitos profesionales y administrativos, el desarrollo y generalización de estos objetivos. El entorno de crisis y las características demográficas de nuestro país obligan a que España implemente con decisión tales cambios, de los cuales depende fundamentalmente la sostenibilidad de su sistema sanitario $0^{5,16,21}$.
En este sentido, ya hay estudios económicos de salud que concluyen que por ej. el envejecimiento no es tanto una amenaza para los sistemas sanitarios, como una oportunidad para resolver problemas de la gestión de la asistencia sanitaria. En la ordenación de esta deben abordarse problemas de naturaleza política, institucional, corporativa o económica en relación con decisiones y objetivos del propio sistema sanitario. En la solución de tales problemas influirán además de la evolución demográfica el modo de enfocar cuestiones como la solidaridad intergeneracional, la edad de jubilación, la tasa de natalidad, el nivel de ocupación o la formación, etc. ${ }^{21}$.

\section{La Situación de partida en Galicia y la Estrategia SERGAS 2014}

Con respecto a Galicia, en el capítulo final de este Monográfico se detallarán pormenorizadamente los objetivos y líneas estratégicas contenidos en la Estrategia SERGAS 2014, La Sanidad Pública al servicio del paciente. En lo que se refiere a dichas las enfermedades crónicas, la radiografía de partida definida en dicho documento -con algunos datos actualizados a 2010- es la siguiente 22 :

1. Valoración de la Sanidad. Según el Barómetro Sanitario del Ministerio de Sanidad, la sanidad pública en Galicia es la peor valorada (sólo por detrás de Canarias). Todos los aspectos referidos a la atención que se presta en las consultas de medicina general son peor valorados en Galicia. Es la CA con mayor \% de usuarios que afirman tener que esperar $>6$ meses para acudir al especialista (21\% frente $7 \%$ nacional). A 31/03/2009 el tiempo medio de espera para pacientes con prioridad 1 era 40,2 días.

2. Estado de salud. Percepción de su estado de salud peor que el resto de CCAA. Opina que su estado de salud es regular ( $31,1 \%$ frente a $21,8 \%$ de media), malo $(8,2 \%$ frente a $5,8 \%$ ) o muy malo (2,5\% frente a $1,7 \%)$. Se ha producido un incremento de patologías crónicas y de pacientes pluripatológicos. Galicia presenta una mayor incidencia de enfermedades del aparato respiratorio, circulatorio y neoplasias malignas que la media.

En la Fig. 1 se resumen los datos actualizados sobre las hospitalizaciones gallegas y su comparación con España (Encuesta de Morbilidad Hospitalaria, 2010)23. Muchos pacientes, sobre todo ancianos, podrán compartir $\geq 2$ de dichas patologías.

3. Demografía. Sufre despoblación progresiva de las zonas interiores de Lugo y Ourense. Presenta grandes zonas de dispersión que coincide con dichas provincias. Las previsiones indican que su población se reducirá un $4 \%$ en los próximos 9 años. Galicia será la $2^{\mathrm{a}} \mathrm{CA}$ (sólo superada por Asturias) con mayor descenso poblacional. Este descenso viene marcado por una tasa bruta de mortalidad $>2,1 \%$ de la media nacional y tasa bruta de natalidad $<3,3 \%$. La 
población gallega está muy envejecida. El 21,2\% es >64 años frente a 18,3\% nacional (INE, 2009). Las provincias más envejecidas son Ourense y Lugo. Ello provocará mayores niveles de sobreenvejecimiento futuros.

4. Gasto sanitario. Como producto de la crisis, el gasto sanitario medio per cápita se redujo en España en 2011 un $4,1 \%$, respecto a 2010. En dicho 2011 el gasto sanitario gallego por habitante fue ligeramente inferior a la media nacional (1266 frente a 1289 ). (Cf. http://www.farodevigo.es/galicia/2011/01/03/galicia-debajo-media-gastosanitario/505602.htm|Galicia).

El gasto farmacéutico per cápita (311 €) es el $3^{\circ}$ más elevado -tras Canarias y Asturias. Este gasto supone $>19 \%$ que la media nacional. Galicia presenta escaso uso de genéricos (4\% frente al $8 \%$ de media) y prácticas ineficientes de prescripción y dispensación. No obstante, con las reformas introducidas en cuanto a prescripción de genéricos y catálogo sanitario se ha observado una mejoría evidente en el último año.

5. Actividad asistencial. La estancia media (2010) en los hospitales gallegos fue de 7,9 días (1,1> media). Galicia tiene un $13 \%<$ Urgencias y un $7,7 \%<$ cirugías que la media. El $n^{\circ}$ de pruebas de Radiodiagnóstico es $>$ que la media (10\% > de radiología convencional, 32\% > TAC y $49 \%>\mathrm{RMN})$.

5. Dotación de recursos. Galicia cuenta con una buena red de centros sanitarios, siendo la dotación de camas y equipamiento en la mayoría de casos superior a la media nacional. La dotación de gammacámaras y PET es menor. Ya en marcha el nuevo hospital de Lugo, se observa retraso en la construcción del nuevo hospital de Vigo.

Los sistemas de información gallegos son potentes y con alto grado de integración.

6. Profesionales. Los profesionales cuentan con un elevado nivel técnico, formación e implicación con el sistema. Sin embargo, se observa cierta falta de motivación en ellos y la existencia de un modelo obsoleto de gestión de personal.

7. Innovación. Existen magníficos profesionales y grupos en investigación biomédica, de trayectoria acreditada. Sin embargo, debe reforzarse la coordinación entre las estructuras y organizaciones relacionadas, en particular los Institutos de Investigación Sanitaria. Faltan mecanismos de integración con la iniciativa del Campus Vida. Se carece de modelos de protección y transferencia de los resultados de la investigación. No se dispone de un sistema integral de iniciativas innovadoras focalizado en los problemas de salud de los ciudadanos, ni espacio de encuentro donde converjan usuarios, centros generadores de conocimiento (Universidades, otros organismos públicos de investigación), empresas biosanitarias y SERGAS.
8. Orientación a ciudadanos y pacientes. No hay suficientes canales de comunicación, participación e interacción con los ciudadanos. No está lo suficientemente potenciada la libre elección de médico y hospital. Existe escaso desarrollo de atención a colectivos específicos: pacientes crónicos, atención sociosanitaria, atención a domicilio, alta resolución, paciente anciano, autocuidados, etc.

9. Gestión. No hay una gestión eficiente de las listas de espera. Existe heterogeneidad de criterios entre centros y no existe una priorización real de patologías. Existen bolsas de ineficiencia en áreas como compras-logística, procesos diagnósticos, gestión energética, etc. Galicia dispone de muchos datos, no traducidos en información útil para gestión y toma de decisiones eficiente.

10. Principales problemas identificados en el Plan de Prioridades (PPS). Los problemas de salud prioritarios identificados por la Consellería en su PPS son: cardiopatía isquémica, cáncer de pulmón, ictus/accidente cerebral vascular, diabetes mellitus, depresión/trastornos afectivos, EPOC, cáncer de colon y mama. Como inciso ha de destacarse aquí la clamorosa falta de referencia a procesos como la insuficiencia cardiaca, con más de 7000 hospitalizaciones anuales.

Además, el PPS identifica los siguientes problemas relacionados con los derechos y procesos: información sobre derechos, deberes y prestaciones; sistema de información para profesionales sanitarios; demora en consultas externas; estancia media en centros públicos; extensión de aseguramiento público a las personas residentes en Galicia; demora para pruebas diagnósticas ambulatorias; escasa cirugía mayor ambulatoria en centros públicos; tiempo de demora para ingreso no urgente; intervenciones quirúrgicas totales por mil habitantes; tasa de cesáreas.

\section{Incorporándose al camino. Los inicios de acción de la Sociedad Gallega de Medicina Interna (SOGAMI)}

La Declaración de Sevilla define como esencial el integrar todos los recursos de la comunidad en la asistencia a tales pacientes, citando específicamente el importante papel de las Sociedades científicas. En Galicia y dentro de la colaboración para desarrollar la Estrategia SERGAS 2014, que intenta indicar pautas de sistematización en el manejo de estos y otros pacientes, la SOGAMI promueve un plan de comienzo para informar e involucrar a sus miembros en una estrategia racional y eficiente en el manejo de pacientes crónicos y pluripatológicos ${ }^{24}$.

Para ello, como objetivos generales se plantea 1. Racionalización de la asistencia a los pacientes PPL proporcionando una atención y cuidados integrales, garantizando la continuidad 
en la asistencia a estos pacientes. 2. Mejorar la accesibilidad evitando las reiteradas visitas y exploraciones en los servicios de urgencias.

Como objetivos específicos desarrollará: 1. Guías de manejo de pacientes pluripatológicos que den soporte a una asistencia y cuidados integrales a través equipos multidisciplinares. 2. Planes terapéuticos interdisciplinares para los perfiles de pacientes pluripatológicos más prevalentes encaminados al tratamiento y cuidado de sus enfermedades. 3. Reuniones periódicas, protocolos compartidos y circuitos con Atención Primaria.

Dicho plan de inicio de la SOGAMI para la Mejora de la Atención a Pacientes Pluripatológicos en Galicia, comprende los siguientes pasos. 1. Conocimiento del punto de partida y divulgación de los planes ya en desarrollo en distintos ámbitos asistenciales del SERGAS. 2. Plasmar dichas experiencias multicéntricas en un Monográfico de Galicia Clínica, revista oficial de la SOGAMI, y difundirlas a través de su Web (www. meiga.info). 3. Elaborar o adaptar guías de manejo de pacientes pluripatológicos para proporcionar cuidados integrados y en lo posible homogéneos a estos pacientes, que incluirán prioritariamente a pacientes con EPOC, IC, dislipemias, HTA, etc. 4. Difundir estas estrategias entre los miembros de la Sociedad a través de su Revista y página Web específicas así como a través de la red informática del SERGAS. 5. Divulgar e implementar dichas Guías de actuación por los profesionales mediante su adaptación a los diferentes ámbitos locales. Para ello, y como parte de este plan estratégico de desarrollo de tal proyecto ya se firmó en diciembre-2011 un convenio no condicionado con la empresa AstraZeneca y se exploran otras valiosas colaboraciones de otras empresas e instituciones.

El presente Monográfico de Galicia clínica es el inicio de la materialización de este proyecto y planea distribuirse a los asistentes del IV Congreso Nacional de Atención al paciente crónico (marzo-2012), a los miembros de SOGAMl y a órganos competentes del SERGAS. Completada la $1^{\text {a }}$ fase de elaboración del Monográfico, se pasará a una $2^{\mathrm{a}}$ de elaboración de actuaciones clínicas para el paciente crónico/pluripatológico, se diseñarán estudios epidemiológicos en el SERGAS para la identificación y asistencia de pacientes con múltiples reingresos hospitalarios, se incorporarán en su Web protocolos de atención de las Unidades de Cuidados Paliativos, etc.

En conclusión, creemos que las sociedades científicas, como la SOGAMI, deben mostrarse sensibles y deseosas de aportar y colaborar en la mejor asistencia a este tipo de pacientes complejos y cada vez más prevalentes. Para ello se han iniciado planes de sensibilización entres su miembros y se desarrollan pautas de actuación homogeneizadas para el manejo de tales pacientes.

\section{Bibliografía}

1. Ollero Baturone M. Modelo de organización para los pacientes crónicos complejos. Foro de Debate. Gestión clínica 2.0. Escuela andaluza de Salud Pública, 2009.

2. Ollero-Baturone M (coordinador), Álvarez-Tello M, Barón-Franco B, Bernabeu-Wittel $\mathrm{M}$, et al. Atención al paciente pluripatológico: Proceso Asistencial Integrado. $2^{\mathrm{a}}$ ed. Sevilla: Consejería de Salud; 2007. Available at: http://www-csalud.dmsas.sda.sas. junta-andalucia.es/contenidos/procesos/docs/pluri.pdf.

3. Ollero M, Bernabeu M, Rincón M, Upshur R, Bernstein B. The language of polypathology. En: Jadad AR, Cabrera A, Martos F, Smith R, Lyons RF. When people live with multiple chronic diseases: a collaborative approach to an emerging global challenge. Granada: Andalusian School of Public Health; 2010. Available at: http://www.opimec. org/equipos/when-people-live-with-multiple-chronic-diseases.

4. Ribera Casado M. La salud y las personas mayores. En Libro blanco del envejecimiento ativo. Ministerio de Sanidad, Política Social e lgualdad: Madrid; 2011; pp. 225-45.

5. Ministerio de Sanidad y Consumo. Unidad de Pacientes Pluripatológicos. Estándares y Recomendaciones. 2009. Disponible en: www.msc.es.

6. Fernández Miera MF. Atención al paciente pluripatológico en HaD. En: Sanroma Mendizábal P, Sampedro García I, González Fernández C, Baños Canales MT. Hospitalización domiciliaria. Recomendaciones clínicas y procedimientos. Sociedad española de hospitalización a domicilio. Santander: Imprenta Regional de Cantabria, 2011, 419-427.

7. Ruiz de Adana Pérez R. Paciente con enfermedades crónicas: ¿cómo mejorar su atención? 2011. Disponible en: http://sedisasigloxxi.es/spip.php?article128.

8. García-Morillo JS, Bernabeu-Wittel M, Ollero-Baturone M, Aguilar-Guisad M, RamírezDuque N, González de la Puente MA et al. Incidencia y características clínicas de los pacientes con pluripatología ingresados en una unidad de medicina interna. Med Clín (Barc) 2005; 125: 5-9

9. Ramírez-Duque N, Ollero-Baturone M, Bernabeu-Wittel M, Rincón-Gómez M, OrtizCamúñez M.A, García-Morillo JS. Características clínicas, funcionales, mentales y sociales de pacientes pluripatológicos. Estudio prospectivo durante un año en Atención Primaria. Rev Clin Esp. 2008; 208(1):4-11.

10. Zambrana García JL, Velasco Malagon MJ, Diez García F, Cruz Caparros G, Martín Escalante MD,Adarraga Cansino MD. Characteristics of patients with multiple disease hospitalized in Internal Medicine services. Rev Clin Esp. 2005; 205(9):413-417.

11. Medrano González F, Melero Bascones M, Barba Romero MA; Gómez Garrido J, Llabrés Díaz J; Moreno Salcedo J. Comorbilidad, pluripatología, consumo de recursos y pronóstico de pacientes ingresados en una unidad de Medicina Interna. An Med. Intern. (Madrid) 2007; 24: 525-530.

12. Martínez Velilla NI, De Gaminde Inda I. Índices de comorbilidad y multimorbilidad en el paciente anciano. Medicina Clínica. 2011; 136(10): 441-446.

13. Sociedad Española de Medicina Interna(SEMI). [Web site]. Madrid: SEMl. Grupos de Trabajo FEMI. Pacientes Pluripatológicos y Edad Avanzada. Disponible en: http://www. fesemi.org/grupos/edad_avanzada/noticias/index.php.

14. Bernabeu-Wittel M, M. Ollero-Baturone M, Moreno-Gaviño L, Barón-Franco B, Fuertes A, Murcia-Zaragoza $\mathrm{J}$ et al. Development of a new predictive model for polypathological patients. The PROFUND index. Eur J Intern Med (2010), doi:10.1016/j. ejim.2010.11.012

15. Nuño Solinís R. Atención innovadora. Más necesaria que nunca. RISAI 2009; 1(3): 1-8. disponible en www.risai.org.

16. Sociedad Española de Directivos de Atención Primaria. Un nuevo hospital para una nueva realidad. Saned: Madrid, 2011. Disponible en www.sedap.es.

17. Observatory of Innovative Practices for Complex Chronic Diseases Management. [Web site].Taxonomy. Available at: http://www.opimec.org/glosario.

18. Grupo de Trabajo de la Sociedad Española de Medicina Interna (SEMI) y la Sociedad Española de Medicina Familiar y Comunitaria (semFYC). Atención al paciente con enfermedades crónicas Documento de consenso. Sevilla: Mergablum Edición y Comunicación SL, 2011.

19. Observatorio de prácticas innovadoras en el Manejo de Enfermedades Crónicas Complejas. Disponible en www.opimec.org.

20. Ollero Baturone M, Orozco Beltrán D, Domingo Rico C, Román Sánchez P, López Soto A, Melguizo Jiménez M, Bailón Muñoz E, Ruiz Cantero A, Ortiz Camúñez MA, Cuello Contreras JA. "Declaración de Sevilla" conferencia nacional para la atención al paciente con enfermedades crónicas. Rev Clin Esp. 2011; 211: 604-6.

21. Hidalgo Vera A, coord. Estudio de las pautas previsibles de utilización futura de servicios sanitarios por mayores, frene a la viabilidad financiera del sistema de salud. Fundación Pfizer: Madrid, 2011. Disponible en www.fundazionpfizer.org.

22. Estrategia SERGAS 2014. La Sanidad Pública al servicio del paciente. Disponible en: http://www.sergas.es/Docs/Conselleria/Estrategia_Sergas_2014.pdf,consultado, 30/01/2011.

23. Instituto Gallego de Estadística, 2012. Encuesta de Morbilidad hospitalaria, 2010.

24. Montes-Santiago J, Casariego-Vales E, De Toro Santos M , De La Iglesia Martínez F, Cerqueiro JM, Mosquera, Grupo SOGAMI. Mejora de la atención al paciente crónico y pluripatológico en Galicia. Plan de inicio de la Sociedad Gallega de Medicina Interna. IV Congreso Nacional de Crónicos, Alicante, marzo 2012. 